\title{
Evaluation of Urinary Oxidative stress Marker and presence of microalbuminuria in patients with Covid-19 disease as a measure of kidney involvement \\ *Sadq A.Frayh \\ **Dr.Mohammed AbdulateefAlbayati \\ $* * *$ Dr. Nawar S.Jamil \\ */ Al-Nahrain University /College of Medicine / Department of Chemistry and Biochemistry \\ **/ Al-Nahrain University /College of Medicine / Department of Chemistry and \\ Biochemistry \\ ***/ Al-kindy Teaching Hospital \\ E.mail :mustafasaleamuob@gmail.com \\ Mobile : +9647717419551
}

\begin{abstract}
subject :COVID19 virus, as of those being pandemic, infected millions of humans dead and because of the unknown impact of the pandemic on the population .in Wuhan is a new coronavirus discovered in China in December 2019. By WHO, it is predicted that, by March 11, 2020, the disease pandemic will have spread throughout the world. Must patient was found elevated D- Dimer, oxidative stress reactive oxygen species (ROS), and fasting blood sugar. Besides, increase blood urea and end with dialysis.

objective of the study: To better understand coronavirus disease 2019 (COVID-19) by finding possible predictors for kidney damage by a correlation between microalbuminuria and albumin creatinine ratio (ACR)with oxidative stress marker 8-epi-prostaglandin F2alpha in urine

\section{Material and method :-}

group I; consist 80 participant (male \&female ) hospitalization with covid 19 sever and non sever (confirmed by polymerase chain reaction) COVID-19 patients showed a significant elevation in the levels of albuminurea and oxidative stress marker , we concluded a proportional correlation between the aforementioned parameters and COVID-19 suggesting the uses of these tests to the diagnosis early kidney damage
\end{abstract}

-group II : consist 80 participant (male \&female ) recovering from corona virus as acontrol 
Results :This study showed high correlation between of Albuminurea and albumin creatinin ratio (ACR) with oxidative stress marker8-epi-prostaglandin F2alpha in non-sever ans sever in patients with COVID-19. Also the study showed high correlation between D-dimer and disease progression and severity in patients with COVI19.

Conclusion :There is correlation between of Albuminurea and albumin creatinin ratio (ACR) with oxidative stress marker8-iso -prostaglandin F2alpha in non-sever ans sever in patients with COVID-19 during Progression

Keywords : COVID-19, Severe Acute Respiratory Syndrome (SARS), ACR oxidative stress , 8-epi-prostaglandin F2alpha lipid peroxidation and albuminurea

\section{Introduction}

when the disease develops because the cells of the immune system are impaired, and their ability to deal with oxidative stress is reduced, leading to excessive oxidative damage which will instead During viral infection, oxidative activity, the lymphocytes will have a more altered structure and function, for example, CD4 (decreased, disintegrated) cells are likely to be found. The condition inside the cells could be a source of oxidative stress, leading to cell membrane weakening and decreasing reactivity and viability(1). Oxidative stress actually influences cell death, the process in which cell death is initiated. apoptosis may be a mechanism implicated in CO19 therapy. Here a subject's supply of H2O2, at the onset of infection, is detected. Scientists think that they observed higher levels of H2 in the COvid19 patients, a condition in which innate immunity-damaged immune cells go out of control, and then hypothesize that the oxidized and immune cells are connected (1). one of the major contributing factors of COVID-19-related death . Similar to SARS-CoV and MERS, the kidneys are potential targets for COVID-19 .Podocytes and proximal tubular epithelial cells, highly enriched with ACE 2 receptors, are distinctive targets for SARS-CoV-2 (2). In a multi-centered, retrospective, observational study in two hospitals in Wuhan. China, more than (28\%) of the cohort developed AKI. Proteinuria, hematuria, increased blood urea nitrogen levels, and serum creatinine were present in $10 \%$ of patients, respectively. These variables, along with urine analysis and D-dimer, were significantly associated with the death of COVID-19 patients. The existence of microalbumin, $\alpha 1$-microglobulin, immunoglobulin-G, and transferrin in urine analysis of most patients confirms glomerular and tubular injury associated with COVID-19 infection (3). A recent systematic review and meta-analysis have reported that the overall incidence of AKI in all COVID-19 patients was 4.5\%. In mild or moderate cases, severe cases and critical cases of COVID-19, , AKI 
appears to be closely associated with both the severity and prognosis of COVID-19 patients . It was determined by histopathology from patient renal tissue that SARS2 targets

the renal tubules directly through the systemic circulation. Therefore, consequently endothelial cells mediated macrophage and tubular nephrocyte phagocytosis fibrosis causes further tissue damage (35). drinking less water than is required They contain medications that are toxic to the kidneys, such as non-steroidal antiinflammatory drugs (NSAIDs),

\section{Patients, Materials and Methods:}

\section{Patients:}

\section{Patients and study design:}

\section{n2.1 .subject and study protocol}

This case control study was conducted from December 2020 to march 2021 at the department of Chemistry and Biochemistry , College of Medicine AL-Nahrain university on 80 patient with covid 19 divided to sever and non sever in ALKindy Teaching Hospital comparing with 80 age and gender - matched participants as a control group each participant in this study was asked to acceptance consent showing their agreement for participation in this study. meets any of the following criteria as determined by the Iraqi Ministry of Health guidelines: appendixC

Respiratory distress ( $\mathrm{RR}>$ than 30 / $\mathrm{m}$ in adults)

Blood oxygen saturation lees than $93 \%$ on ambient air.

Lung infiltrates $>50 \%$ of the lung field within 24 hours. Blood oxygen saturation lees than $93 \%$ on ambient air. Lung infiltrates $>50 \%$ of the lung field within24 hours. Participant in this study and divided tow group groups:-group I; consist 80 participant (male \&female ) hospitalization with covid 19 divided tow sub group groups sever and non sever

-group II : consist 80 participant (male \&female ) recovering from corona virus as acontrol 


\section{Inclusion criteria}

Patients both sexes age less than 65 years admitted to ( COVID-19 care ALKindy Teaching ), who tested positive for SARS-CoV-2 by nasopharyngeal and/or pharyngeal swab

\section{Exclusion criteria}

1- chronic disease

2- Pregnant women

3- Above 65years old individuals

4- A patient infected with SARS-CoV-19 urinates through Foley Blood sample collection and storage:

All the sample (urine \&blood )collected in AL-Kindy Teaching Hospital in Epidemiological lobbies (isolation)

About $5 \mathrm{ml}$ of blood sample and $10 \mathrm{ml}$ off urine taken from 80 patient male and female that hospitalization in and the Kindy Teaching Hospital with covid-19 between severe and non-severe state both sex and the same volume of sample collection from healthy people as a control

(10 ml )of urine centrifugation about 10-minutes at 3 thousand rotation per minute (RpM)before analyzed of oxidative stress marker 8-epi-prostaglandin F2alpha by ELIZA

assay and to measurement albumin and creatinine ratio

( $5 \mathrm{ml}$ ) of blood divided to tow volume $3 \mathrm{ml}$ in Jel tube to measurement of the lipid profile and renal function and liver function and leave in room temperature about 10 minutes before centrifugation at 3000rpm and second volume of of blood tow $\mathrm{ml}$ in sodium citrate tube to measurement D-dimer that mix well and centrifugation 10-minutes at $3000 \mathrm{RpM}$

Result

Non-smoker was the most frequently seen Smoking status ( $n=60.6 \%)$.

For the gender the most frequently observed category was male ( $n=78,54 \%)$. Frequencies and percentages are presented in Table1-1.

Table1-1 :Show the percentage of smoking frequency in study individuals

\begin{tabular}{|c|c|c|c|}
\hline & & Count & Percent \\
\hline \multirow[t]{2}{*}{ smoking } & smoker & 63 & $39.4 \%$ \\
\hline & non smoker & 97 & $60.6 \%$ \\
\hline \multicolumn{2}{|l|}{ Overall } & 160 & $100.0 \%$ \\
\hline
\end{tabular}


Table 1-2show Correlation between groups control ,non-severe and severe for main marker oxidative stress lipid peroxidation 8-epi-prostaglandin F2alpha (8iso-PGF2 $\alpha$ ) and Urin albumin Observations high significant of $P$ value $\geq 0.05$

\begin{tabular}{|l|c|c|c|c|}
\hline \multirow{3}{*}{ Variables } & \multicolumn{3}{|c|}{ Mean \pm Standard deviation } & \\
\cline { 2 - 5 } & Control & Non-severe & Severe & $\begin{array}{c}\text { ANOVA } \\
\text { P value }\end{array}$ \\
\hline U.Alb & $22.53 \pm$ & $280.62 \pm 107.5$ & $279.5 \pm$ & $<0.001$ \\
& 5.56 & & 85.1 & \\
\hline 8-isopsg & $72.7 \pm 17.0$ & $519.2 \pm 206.2$ & $692.6 \pm$ & $<0.001$ \\
& & & 302.4 & \\
\hline
\end{tabular}

U.Alb:- Urinalbumin , 8-isopsg main marker oxidative stress lipid peroxidation 8-epi-prostaglandin F2alpha (8-iso-PGF2 $\alpha$ )

Table 1-3

Correlation between groups control ,non-severe and severe for age ,fasting blood sugar ,renal function , and albumin creatinin ratio, D-dimer we observation high significant correlation between groups and $p$ value $\geq 0.05$

\begin{tabular}{|c|c|c|c|c|}
\hline \multirow[b]{2}{*}{ Variables } & \multicolumn{3}{|c|}{ Mean \pm Standard deviation } & \multirow[b]{2}{*}{$\begin{array}{l}\text { ANOVA } \\
\text { P value }\end{array}$} \\
\hline & Control & Non-severe & Severe & \\
\hline Age & $48.62 \pm 9.77$ & $49.49 \pm 10.13$ & $50.28 \pm 13.41$ & 0.57 \\
\hline FBS & $\begin{array}{c}104.69 \pm \\
12.75 \\
\end{array}$ & $135.05 \pm 49.37$ & $180.28 \pm 71.61$ & $<0.001$ \\
\hline Urine Cr & $215.5 \pm 33.4$ & $143.5 \pm 92.5$ & $38.88 \pm 36.7$ & $<0.001$ \\
\hline Creatinine & $0.82 \pm 0.21$ & $0.75 \pm 0.158$ & $1.47 \pm 0.46$ & $<0.001$ \\
\hline ACR & $0.11 \pm 0.03$ & $4.49 \pm 4.91$ & $15.53 \pm 9.83$ & $<0.001$ \\
\hline urea & $35.47 \pm 5.78$ & $40.41 \pm 9.19$ & $108.07 \pm 83.13$ & $<0.001$ \\
\hline D-dimer & $0.09 \pm 0.09$ & $1.4 \pm 0.5$ & $2.16 \pm 0.36$ & $<0.001$ \\
\hline
\end{tabular}

FBS:- Fasting blood sugar ,Urincr :- Urine creatinin , ACR :- Albumin creatinin ratio, 


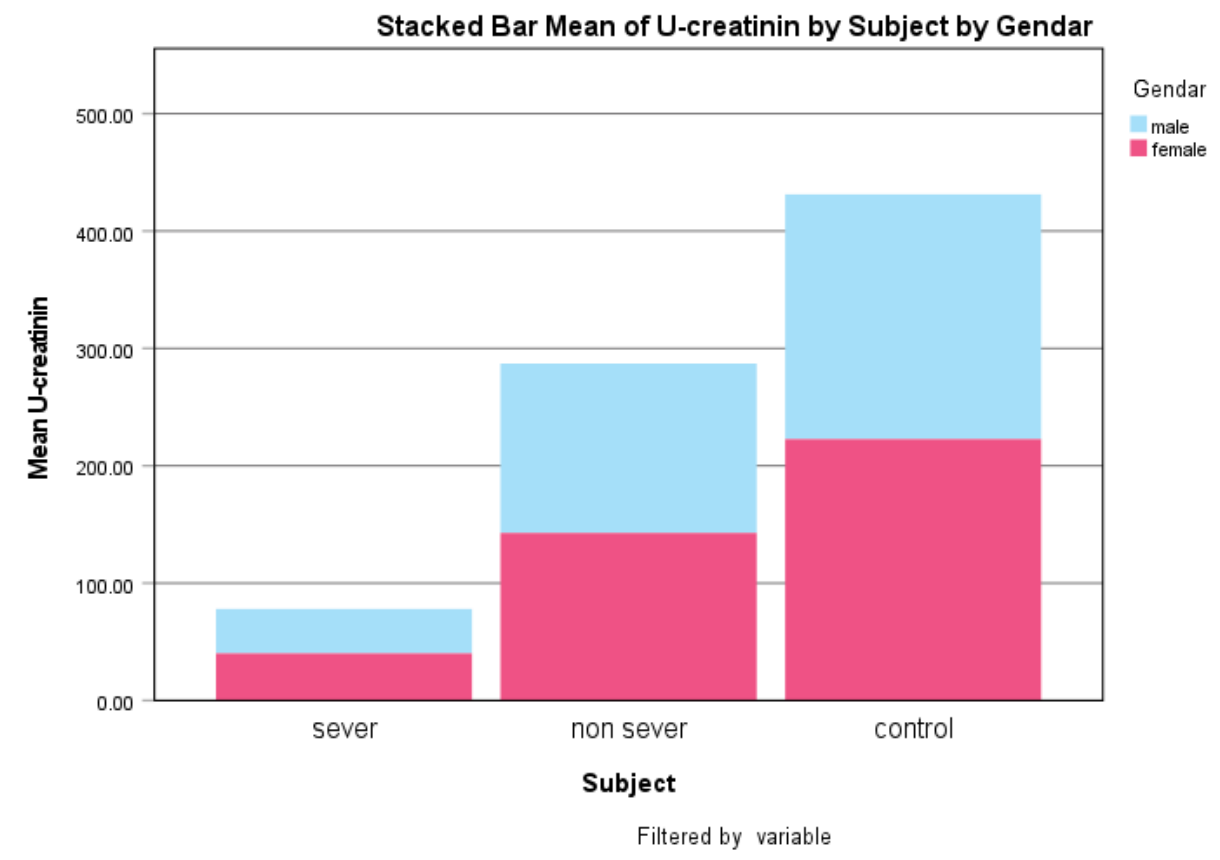

Figure 1-1 Bar chart of urinalbumin concentration in covid 19 patient by severity groups to both gender

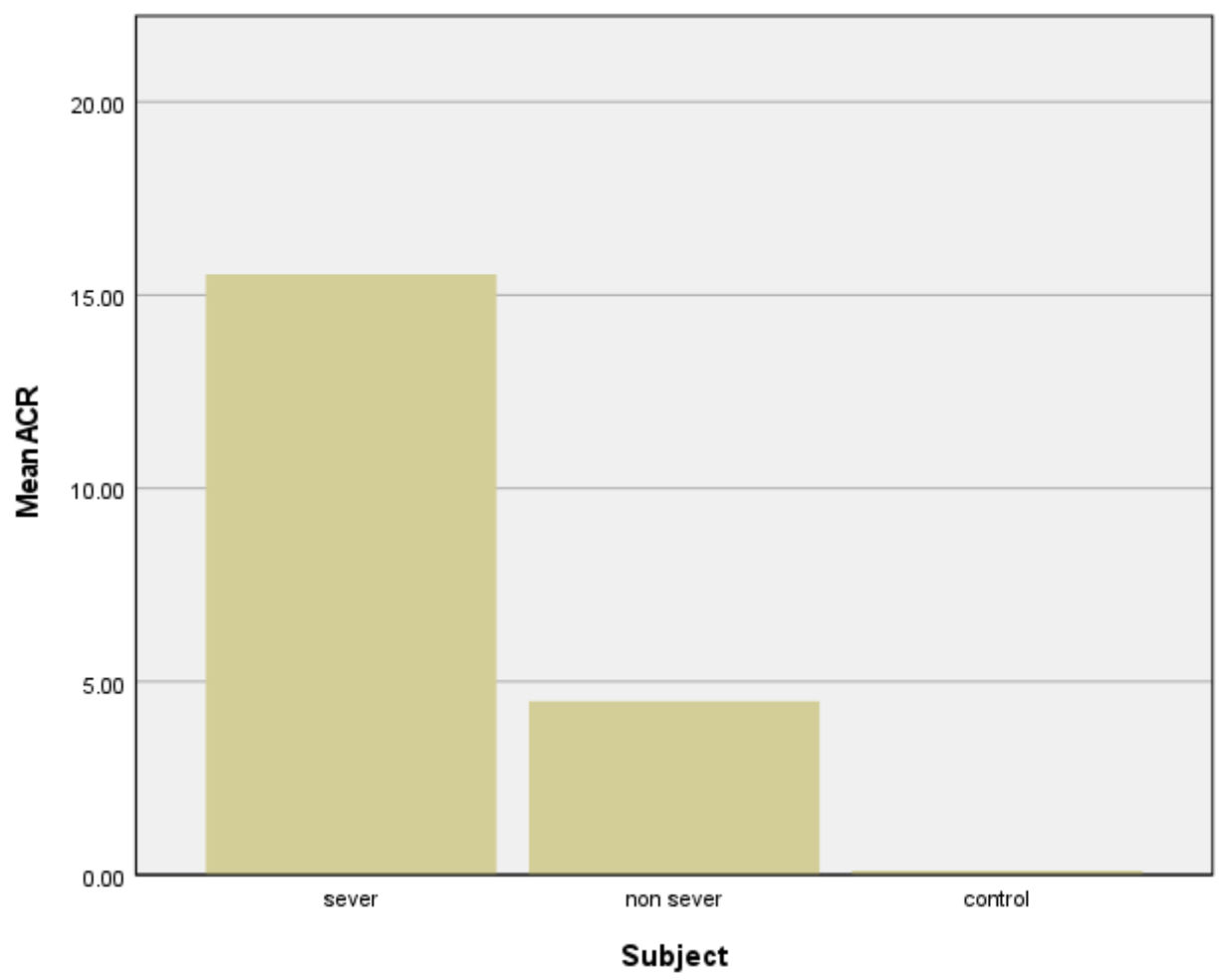

Figure 1-2 Bar chart of ACR in covid 19 patient by severity groups 


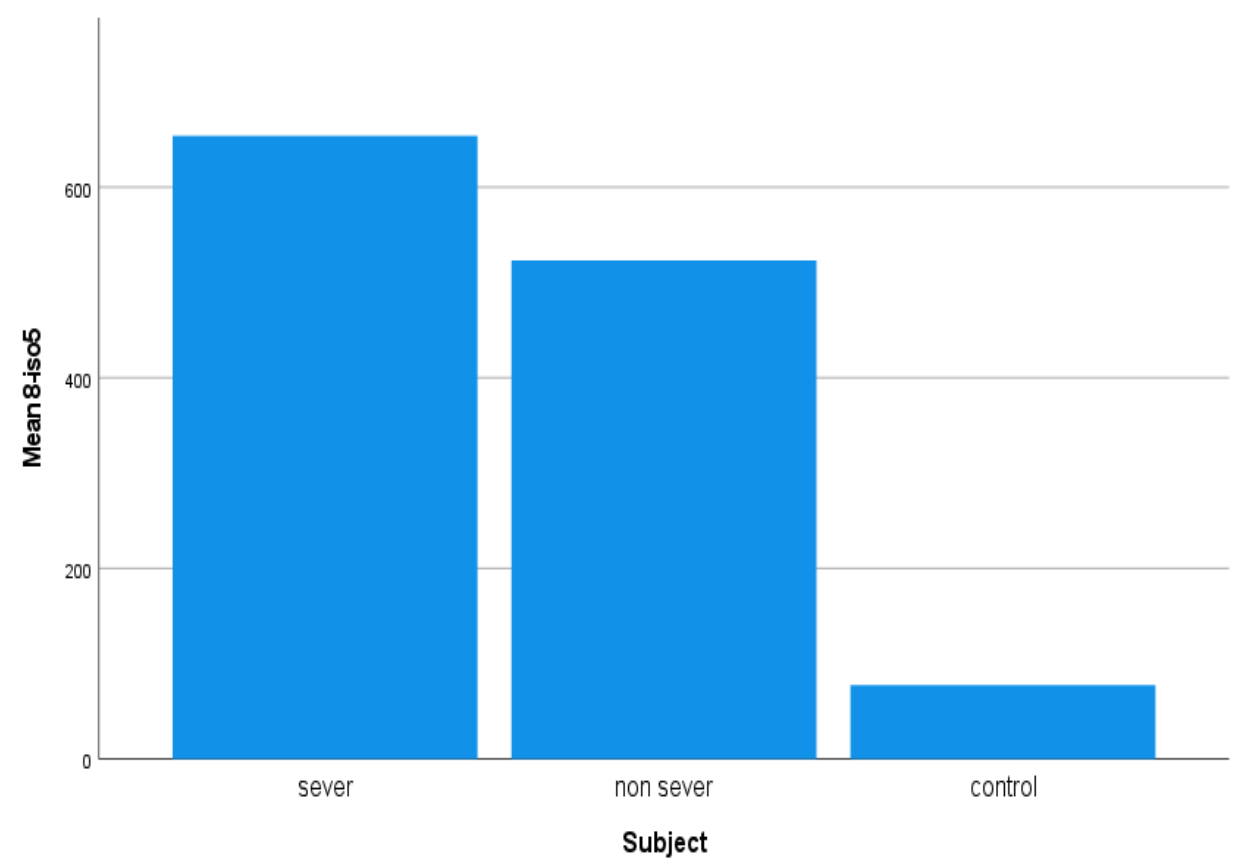

Figure 1-3 Bar chart of oxidative stress marker8-iso -prostaglandin F2alpha in covid 19 patient by severity groups

\section{Discussion}

the information to the covid-19 was a limitation, but we know the development of the disease in shortening made injury with mast cell of the organ body and increase turbidity and mortality human in the world most organ failure that causes and development of the disease was heart failure and renal injury and end with renal failure besides that attack on the brain and nervous system and cause many problems and there and they are nervous system

covid-19 development causes infertility and reduces the endocrine secretion of hormones, and attack the immunity to made cytokine storm that's maybe leading cause of increased mortality in the covid-19

what's the main reason and the dead people infection with this disease was in pulmonary embolism and lung damage that reduction of oxygen supply to the organ and hypoxia resulting death of the patient

in the present study, the kidney involvement by the disease are studied by measuring urine creatinine, urine albumin, Albumin creatinin ratio and urea that combined with oxidative stress biomarker 8-iso-PGF2 $\alpha$, .which reflect kidney damage 
We show in this study There is a low correlation significant Difference With covid-19 infection that was established in table number 3-4where p-value to the 0.57 But we offer the severity of the case older age then none severe that have an average equal to50.28 \pm 13.25 besides the smoking We show the figure 3-11 that smokes are patient more severity and death than their non-smoker patient(5) 8-iso-PGF $2 \alpha$ oxidative stress for lipid peroxidation high correlation between groups,

Average for non-severe $519.2 \pm 206.2$ average for severe $692.6 \pm 302.4$ and for control $72.7 \pm 17.0 \quad \mathrm{P}$-value $=<0.001$ in this study we evaluate renal damage during covid19 viral infection by the correlation between oxidative stress marker 8-iso PSG lipid peroxidation and Microalbuminuria, Albumin creatinine ratio (ACR), to discussion mild or non-severe case in the hospitalization in the table high correlation coefficient between $\mathrm{D}$-dimer $\mathrm{r}=0.567$ and $\mathrm{p}$-value $=30.0001$ and oxidative stress marker in same table show high correlation coefficient between oxidative stress marker and albumin creatinine ratio ACR that mean in the same time where are be elevated oxidative stress that resulting from thrombin and deep vein thrombosis to elevated D-dimer concentration that means in Direct correlation reduced glomerular filtration rate and increase secretion of albumen in the urine important this information in the non-severe case with covid-19 infection that got lion to early prognosis kidney damage to early treatment antioxidant to reduce oxidative stress ROS to prevent severity continue to the severe or critical state in table 3 -7 high correlation betowen D-dimer and 8-isoPGF $2 \alpha \quad r=0.406$ and $p$ - value $=0.007$ that statisticly strong correlation but less than non-severe so I suggest because high dose of antioxidants as vitamin , Trace metal and anticoagulant drugs

Conclusion:There is correlation between of Albuminurea and albumin creatinin ratio (ACR) with oxidative stress marker8-iso -prostaglandin F2alpha in non-sever ans sever in patients with COVID-19 during Progression

reference

1- Günther M, Al Nimer F, Piehl F, Risling M, Mathiesen T. Susceptibility to oxidative stress is determined by genetic background in neuronal cell cultures. eNeuro. 2018;5(2)

2- Lu W, Kang J, Hu K, Tang S, Zhou X, Yu S, et al. Angiotensin-(1-7) inhibits inflammation and oxidative stress to relieve lung injury induced by chronic intermittent hypoxia in rats. Brazilian J Med Biol Res. 2016;49(10):

3- . Gorbalenya AE, Baker SC, Baric RS, de Groot RJ, Drosten C, Gulyaeva AA, et al. The species Severe acute respiratory syndrome-related 
coronavirus: classifying 2019-nCoV and naming it SARS-CoV-2. Nat Microbiol. 2020;5(4):536-44.

4- Wang LF, Shi Z, Zhang S, Field H, Daszak P, Eaton BT. Review of bats and SARS. Emerg Infect Dis. 2006;12(12):1834-40.

5- Rabb H, Rabb H. patient care Kidney diseases in the time of COVID-19 : major challenges to patient care. 2020;130(6):2749-51. 